\title{
Variations of topside ionospheric scale heights over Millstone Hill during the 30-day incoherent scatter radar experiment
}

\author{
L. Liu ${ }^{1}$, W. Wan ${ }^{1}$, M.-L. Zhang ${ }^{1}$, B. Ning ${ }^{1}$, S.-R. Zhang ${ }^{2}$, and J. M. Holt ${ }^{2}$ \\ ${ }^{1}$ Institute of Geology and Geophysics, Chinese Academy of Sciences, Beijing 100029, China \\ ${ }^{2}$ Haystack Observatory, Massachusetts Institute of Technology, Westford, MA, USA
}

Received: 22 May 2007 - Revised: 11 August 2007 - Accepted: 18 September 2007 - Published: 2 October 2007

\begin{abstract}
A 30-day incoherent scatter radar (ISR) experiment was conducted at Millstone Hill $\left(288.5^{\circ} \mathrm{E}, 42.6^{\circ} \mathrm{N}\right)$ from 4 October to 4 November 2002. The altitude profiles of electron density $N_{e}$, ion and electron temperature ( $T_{i}$ and $T_{e}$ ), and line-of-sight velocity during this experiment were processed to deduce the topside plasma scale height $H_{p}$, vertical scale height VSH, Chapman scale height $H_{m}$, ion velocity, and the relative altitude gradient of plasma temperature $\left(\mathrm{d} T_{p} / \mathrm{dh}\right) / T_{p}$, as well as the $F_{2}$ layer electron density $\left(N_{m} \mathrm{~F}_{2}\right)$ and height $\left(h_{m} \mathrm{~F}_{2}\right)$. These data are analyzed to explore the variations of the ionosphere over Millstone Hill under geomagnetically quiet and disturbed conditions. Results show that ionospheric parameters generally follow their median behavior under geomagnetically quiet conditions, while the main feature of the scale heights, as well as other parameters, deviated significantly from their median behaviors under disturbed conditions. The enhanced variability of ionospheric scale heights during the storm-times suggests that the geomagnetic activity has a major impact on the behavior of ionospheric scale heights, as well as the shape of the topside electron density profiles. Over Millstone Hill, the diurnal behaviors of the median VSH and $H_{m}$ are very similar to each other and are not so tightly correlated with that of the plasma scale height $H_{p}$ or the plasma temperature. The present study confirms the sensitivity of the ionospheric scale heights over Millstone Hill to thermal structure and dynamics. The values of $\mathrm{VSH} / H_{p}$ tend to decrease as $\left(\mathrm{d} T_{p} / \mathrm{dh}\right) / T_{p}$ becomes larger or the dynamic processes become enhanced.
\end{abstract}

Keywords. Ionosphere (Ionospheric disturbances; Midlatitude ionosphere; Plasma temperature and density)

Correspondence to: L. Liu

(liul@mail.iggcas.ac.cn)

\section{Introduction}

The ionospheric scale height, as a measure of the shape of electron density $\left(N_{e}\right)$ profiles or the altitude dependence of electron density, is one of the key ionospheric parameters, due to its intrinsic connection to the ionospheric dynamics, plasma temperature and compositions (Luan et al., 2006; Stankov and Jakowski, 2006b). Several definitions of the ionospheric scale heights exist (Liu et al., 2007). In order to facilitate a description, we adopt the following definitions of ionospheric scale heights. The plasma scale height $\left(H_{p}\right)$ is defined as $k_{b}\left(T_{i}+T_{e}\right) / m_{i} g$, where $k_{b}$ is the Boltzmann constant, $g$ the acceleration due to gravity, $m_{i}$ the ion mass, and $T_{i}$ and $T_{e}$ the ion and electron temperatures. The vertical scale height (VSH) is defined as $-\mathrm{d} h / \mathrm{d}\left(\ln \left(N_{e}\right)\right)$, related to the gradient of the measured $N_{e}$ profiles (e.g. Kutiev et al., 2006). The Chapman scale height $\left(H_{m}\right)$ is the scale height used in fitting the $N_{e}$ profiles with the Chapman- $\alpha$ function (e.g. Huang and Reinisch, 1996; Reinisch and Huang, 2004).

Several types of instruments, such as incoherent scatter radars (ISR), ionosondes, radio occultation measurements, in-situ sensors, and Global Positioning System (GPS), have been used to retrieve information on the ionospheric scale heights (e.g. Belehaki et al., 2006; Gulyaeva, 2004; Huang and Reinisch, 1996, 2001; Kutiev et al., 2006; Lei et al., 2005; Reinisch et al., 2004; Reinisch and Huang, 2004; Stankov and Jakowski, 2006a, b). For example, Kutiev et al. (2006) developed a model for the vertical scale height deduced from topside ionosondes. The model has been updated by Kutiev and Marinov (2007). Stankov et al. (2003) proposed a new profile reconstruction method based on simultaneous GPS TEC and ionosonde measurements for deducing the topside ionospheric scale height. Stankov and Jakowski $(2006 \mathrm{a}, \mathrm{b})$ retrieved the topside vertical scale height from radio occultation measurements. Furthermore, Belehaki et al. (2006) made a comparison of the ionospheric scale height for electron density profiles from topside and bottomside

Published by Copernicus Publications on behalf of the European Geosciences Union. 
ionosondes. The diurnal and seasonal variations of $H_{m}$ over Hainan $\left(109.0^{\circ} \mathrm{E}, 19.4^{\circ} \mathrm{N}\right)$ have been reported by Zhang et al. (2006). Furthermore, the seasonal and solar activity features of $H_{m}$ over Millstone Hill $\left(288.5^{\circ} \mathrm{E}, 42.6^{\circ} \mathrm{N}\right)$ have been investigated with ISR observations (Lei et al., 2005) and over Wuhan $\left(114.4^{\circ} \mathrm{E}, 30.6^{\circ} \mathrm{N}\right)$ with digisonde measurements (Liu et al., 2006). They found that $H_{m}$ over Millstone Hill is overestimated by the IRI2001 model (Bilitza, 2001) and the temporal variations of $H_{m}$ can be explained in terms of those in the slab thickness. Liu et al. (2006) reported that a moderate positive correlation is found between $H_{m}$ and the $F_{2}$ layer peak height $\left(h_{m} \mathrm{~F}_{2}\right)$ and a strong correlation between the bottomside thickness parameter $B_{0}$ and $H_{m}$. A common feature is found at 13 global stations; that is, the values of $H_{m}$ are highest in summer and lowest in winter during daytime. It is interesting that, however, the annual variation of $H_{m}$ becomes much weaker or disappears from late night to pre-sunrise. Recently, Liu et al. (2007) conducted a statistical analysis on the diurnal, seasonal, and solar cycle variations of ionospheric scale heights from the 1966-2002 ISR measurements at Arecibo $\left(293.2^{\circ} \mathrm{E}, 18.3^{\circ} \mathrm{N}\right.$; geomagnetic latitude $30^{\circ}$ ), Puerto Rico. Their statistical analysis identified a clear solar activity pattern of VSH, $H_{m}$, and $H_{p}$ over Arecibo. Median values of $H_{m}$ over Arecibo are also found to be highest in summer and lowest in winter during daytime, while it exhibits a much weaker seasonal variation at night. They discussed the similarities/differences and quantitative relationships between these ionospheric scale heights over Arecibo and found that the diurnal behaviors of VSH and $H_{m}$ are much more complicated than that of $H_{p}$.

However, the knowledge of the behavior of the ionospheric scale heights remains insufficient. For example, few works study the storm-time variations of the ionospheric scale heights so far. Fortunately, available accumulated databases of ISR (e.g. Zhang et al., 2004, 2005; Tepley, 1997; Isham et al., 2000), topside sounders (Bilitza et al., 2006) and radio occultation measurements, are extremely valuable data sources for investigating the characteristics of the ionospheric scale heights.

An extended ISR campaign was conducted at Millstone Hill from 4 October to 4 November 2002. This experiment covered both geomagnetically quiet and active periods, providing a unique opportunity to explore the variations of the ionosphere. Combining with the simultaneous observation of EISCAT Svalbard Radar, Zhang et al. (2005) studied the ionospheric variability and found that quasi-periodic oscillations (with periods longer than 1 day) were correlated with neutral composition changes originating from geomagnetic activity. Later, Lei et al. (2006) compared those measurements with the prediction of the IRI2001 model. It is found that IRI2001 is in good agreement with the observed $h_{m} \mathrm{~F}_{2}$ and $f o \mathrm{~F}_{2}$ while it overestimates the topside $N_{e}$ profiles over Millstone Hill.

In this paper, we deduce ionospheric scale heights $\left(H_{p}\right.$, VSH and $\left.H_{m}\right)$, the $F_{2}$ layer peak electron density $\left(N_{m} \mathrm{~F}_{2}\right)$ and height $\left(h_{m} \mathrm{~F}_{2}\right)$, ion velocity, and the altitude gradient of plasma temperature from this ISR observation. The first objective of this analysis is to explore the variations of the ionosphere over Millstone Hill under geomagnetically quiet and disturbed conditions. We will focus on the median behavior of the topside scale heights and their behaviors under geomagnetically quiet and disturbed conditions. Another objective is to investigate the correlations between VSH/Hp and the thermal structure and diffusion processes in the ionosphere over Millstone Hill.

\section{Data source and solar-geophysical conditions}

An ISR experiment was carried out at Millstone Hill from 4 October to 4 November 2002 (Lei et al., 2006; Zhang et al., 2005). The altitudinal profiles of electron density, $N_{e}$, ion and electron temperatures, $T_{i}$ and $T_{e}$, and line-of-sight velocity, $V_{o}$, were provided from the single-pulses and alternating code measurements. $V_{o}$ is essentially the vertical ion velocity $V_{i z}$ during this measurement because the zenith angle of radar beams used is close to the vertical direction $\left(88^{\circ}\right)$. In this study, the single-pulses mode data during this experiment are used for investigation.

Figure 1 illustrates the solar-geophysical conditions during this ISR experiment. The $10.7 \mathrm{~cm}$ solar radio flux $\mathrm{F}_{107}$, a standard solar activity proxy, takes values between 155.1 to 182.5 units $\left(10^{-22} \mathrm{~W} \mathrm{~m}^{-2} \mathrm{~Hz}^{-1}\right)$ during the period from 4 October to 4 November 2002 (day numbers 277-308), while its 81-day average $\mathrm{F}_{107 A}$ is about 174 units. Magnetic indices $\mathrm{AE}, A_{p}$, and $D_{s t}$ are also plotted in Fig. 1 . As can be seen from Fig. 1, three geomagnetic disturbances occurred during this period that caused severe ionospheric effects (Zhang et al., 2005).

When considering the geomagnetic activity effects, a reference level should be selected. There are usually two kinds of reference levels used for the purpose: values from quiet day(s) (21 October for this period), or median values from an extended period (say, 30 days). The median values during this interval served as the reference level for this study.

\section{Analysis and results}

\subsection{Analysis method}

More than 10000 altitudinal profiles of $N_{e}, T_{e}, T_{i}$, and $V_{o}$ were provided from this Millstone Hill ISR experiment. Figure 2 is an example showing the data processing and analysis procedure we adopted. First, median $N_{e}$ profiles are evaluated from raw $N_{e}$ profiles within every 30-min interval in each day. Then $N_{m} \mathrm{~F}_{2}$ and $h_{m} \mathrm{~F}_{2}$ are obtained with a least-squares fitting for the median profiles with a Chapman$\alpha$ function (e.g. Rishbeth and Garriott, 1969),

$$
N_{e}(h)=N_{m} \mathrm{~F}_{2} \exp \{0.5[1-z-\exp (-z)]\},
$$




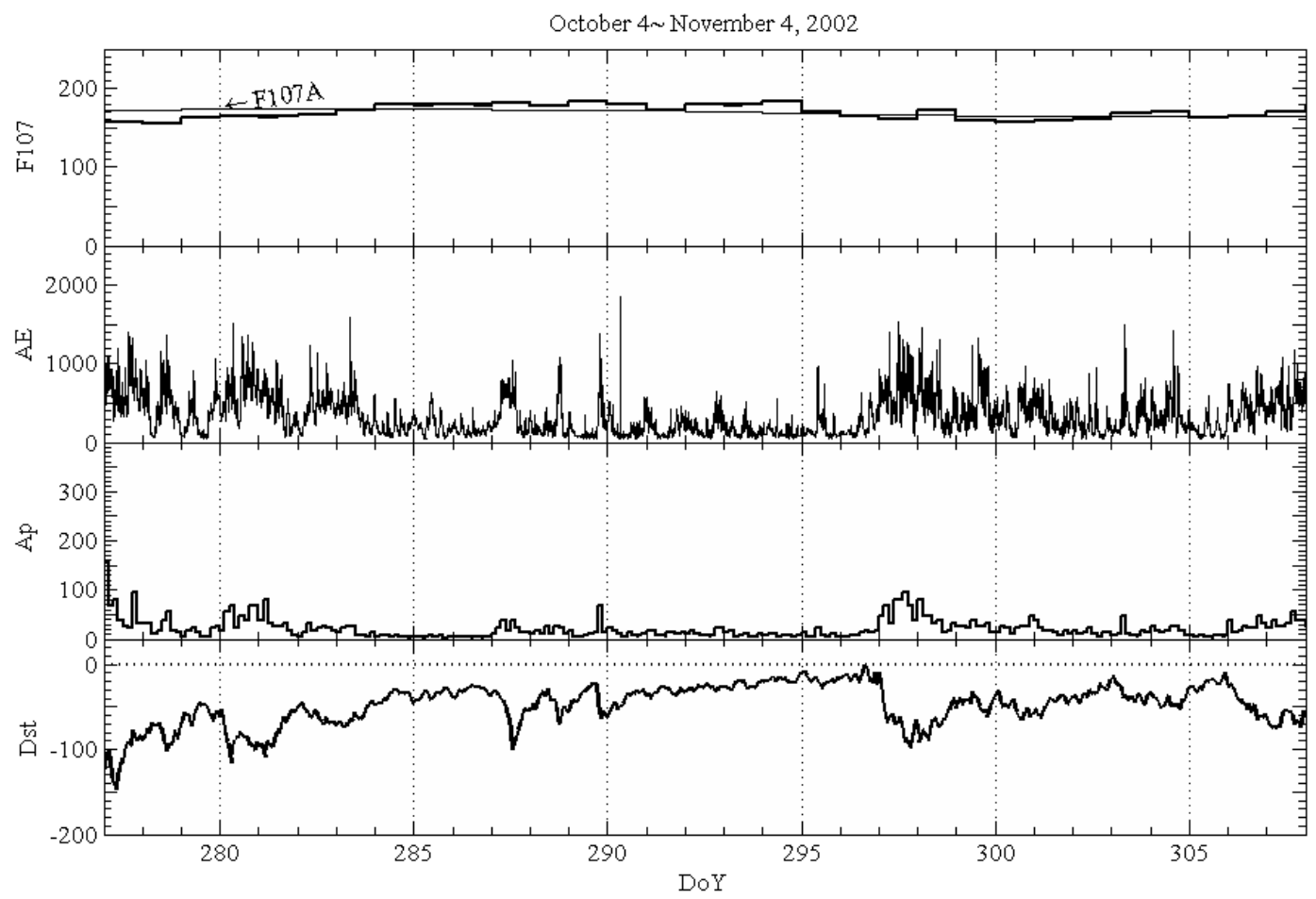

Fig. 1. The solar geophysical conditions during the long-duration Millstone Hill ISR experiment from 4 October and 4 November 2002.

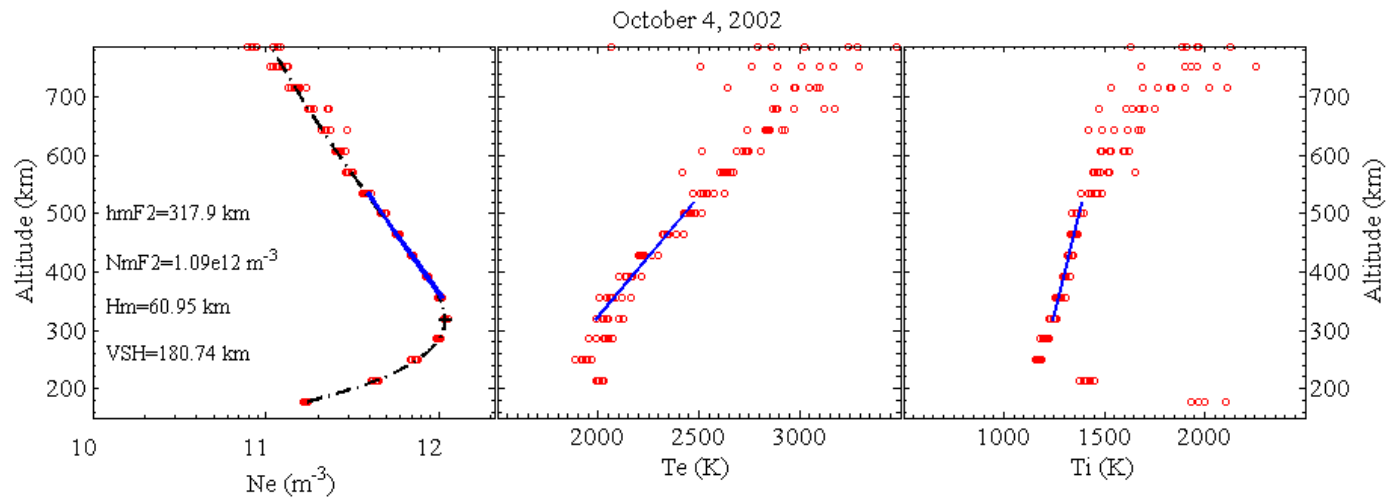

Fig. 2. The Millstone Hill ISR altitude profiles of electron density, $N_{e}$, and ion and electron temperatures, $T_{i}$ and $T_{e}$, during a half-hour interval around 17:50 UT on 4 October 2002. At the left panel, the dashed line shows the fit of $N_{e}$ with a Chapman- $\alpha$ profile function, while the solid line shows the exponential fit. The solid lines at the center and right panels show, respectively, the linear fit for the $T_{i}$ and $T_{e}$ profiles.

$$
z=\left(h-h_{m} \mathrm{~F}_{2}\right) / H(h)
$$

Here the Chapman scale height $H(h)$ is assumed to be of linear dependence on altitude (see Lei et al., 2006; Liu et al., 2007). We denote $H(h)$ at $h_{m} \mathrm{~F}_{2}$ as $H_{m}$ in Eq. (1). $N_{m} \mathrm{~F}_{2}$, $h_{m} \mathrm{~F}_{2}$ and $H_{m}$ are adjustable variables in the least-squares fitting for median profiles, according to Eq. (1).

An example is shown in the left panel of Fig. 2, in which circle points denote the observed $N_{e}$ within a half-hour interval centered at 17:50 UT on 4 October 2002, whereas the dashed curve stands for the fitted result with a Chapman function.

We fit each median $N_{e}$ profile with a Chapman- $\alpha$ function, thus $N_{m} \mathrm{~F}_{2}, h_{m} \mathrm{~F}_{2}$ and $H_{m}$ are determined with the leastsquares fitting procedure. Good fitting prevails in most cases, and we discard those profiles when significant deviations occur, although those profiles may represent the actual situations. Then the values of vertical scale height, VSH, are obtained from the $\left(h_{m} \mathrm{~F}_{2}+25 \mathrm{~km}\right.$ to $\left.h_{m} \mathrm{~F}_{2}+250 \mathrm{~km}\right)$ altitude interval of median ISR $N_{e}$ profiles through a linear fitting for 

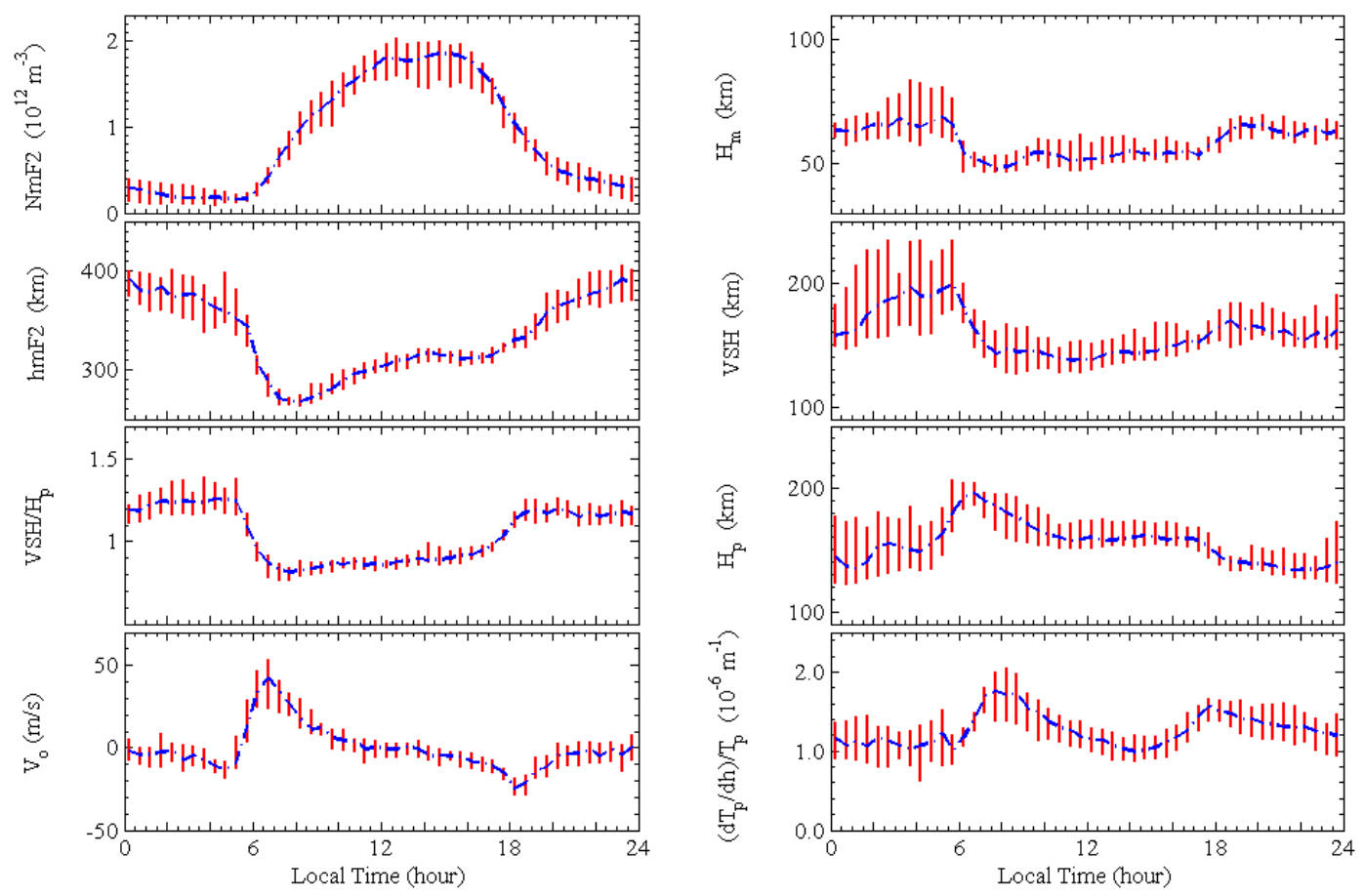

Fig. 3. The diurnal variation of $N_{m} \mathrm{~F}_{2}, h_{m} \mathrm{~F}_{2}$, the line-of-sight velocity $\mathrm{V}_{o}$ (around $500 \mathrm{~km}$ ), the Chapman scale height $H_{m}$, the vertical scale height VSH, plasma scale height $H_{p}$, and the relative gradient of plasma temperatures $\left(\mathrm{d} T_{p} / \mathrm{dh}\right) / T_{p}$ derived from the ISR experiment at Millstone Hill. The ratio of VSH to $H_{p}$ is also plotted. Lines with bars represent, respectively, the half-hourly median values and the corresponding upper (UQ) and lower (LQ) quartiles during the ISR experiment.

$\ln \left(N_{e}\right)$ versus $h$. At the same time, ion and electron temperatures $\left(T_{i}\right.$ and $\left.T_{e}\right)$ and their altitude gradients $\left(\mathrm{d} T_{i} / \mathrm{dh}\right.$ and $\mathrm{d} T_{e} / \mathrm{dh}$ ) at an altitude of $100 \mathrm{~km}$ above the $F_{2}$ peak are also evaluated from a linear fitting $\left(h_{m} \mathrm{~F}_{2}\right.$ to $\left.h_{m} \mathrm{~F}_{2}+200 \mathrm{~km}\right)$ for the observed $T_{i}$ and $T_{e}$ profiles, as shown in the center and right panels of Fig. 2. Thus, $H_{p}$ and the relative altitude gradient of plasma temperature, $\left(\mathrm{d} T_{p} / \mathrm{dh}\right) / T_{p}$, can be easily determined from the fitted $T_{i}$ and $T_{e}$ and their gradients $\left(\mathrm{d} T_{i} / \mathrm{dh}\right.$ and $\left.\mathrm{d} T_{e} / \mathrm{dh}\right) . T_{p}$ is the plasma temperature. Finally, a halfhourly average of ion velocity $V_{o}$ at an altitude of around $500 \mathrm{~km}$ is determined from the raw ISR line-of-sight velocity observations.

\subsection{The median pattern of ionospheric parameters}

A statistically average feature of the ionosphere can be described by the median values of ionospheric parameters. Figure 3 shows the half-hourly median values of parameters $N_{m} \mathrm{~F}_{2}, h_{m} \mathrm{~F}_{2}, H_{m}, \mathrm{VSH}, H_{p}, V_{o}, \mathrm{VSH} / H_{p}$, and $\left(\mathrm{d} T_{p} / \mathrm{dh}\right) / T_{p}$ over Millstone Hill during 4 October to 4 November 2002. Vertical bars in Fig. 3 depict the corresponding upper and lower quartiles values, illustrating the deviations from the average.

Over Millstone Hill, as illustrated in Fig. 3, the variation of the median $N_{m} \mathrm{~F}_{2}$ has a well-defined diurnal pattern, namely with values larger during the daytime than during the night- time. Median $h_{m} \mathrm{~F}_{2}$ has a maximum around midnight, descends slowly after midnight, declines sharply around sunrise, and reaches a minimum at 07:30 LT. Then $h_{m} \mathrm{~F}_{2}$ reverses to increase and reaches its maximum value around midnight. Median $H_{m}$ reaches its maximum at night with values around $65 \mathrm{~km}$ and its minimum during the daytime with values of $50 \mathrm{~km}$. Taking a value of about $160 \mathrm{~km}$ around midnight, VSH increases from midnight till sunrise. Accompanying a sharp sunrise decline, VSH keeps low values during the daytime, although increases slowly in the afternoon and has a second peak at around 18:30 LT. The median values of $H_{p}$ are higher during daytime and lower at night. A significant morning rise is present in $H_{p}$, which is consistent with the morning overshoot of electron and ion temperatures in the $\mathrm{F}$ layer (e.g. Oyama et al., 1996; Sharma et al., 2005). However, the evening peak is not present in $H_{p}$ at Millstone Hill, although it clearly is present in $H_{p}$ at Arecibo (Liu et al., 2007). Overall, our results suggest, over Millstone Hill, that the diurnal behaviors of median VSH and $H_{m}$ are much different from that of $H_{p}$. In other words, density scale heights VSH and $H_{m}$ are not so tightly correlated with the plasma temperature or $H_{p}$.

The ratio $\mathrm{VSH} / H_{p}$ is 1.2 to 1.25 at night and about 0.85 during daytime, displaying a diurnal pattern rather similar with that of $H_{m}$. Accompanying the sunrise descent in $h_{m} \mathrm{~F}_{2}, \mathrm{VSH}, H_{m}$, and the ratio $\mathrm{VSH} / H_{p}$, the peak shifts 

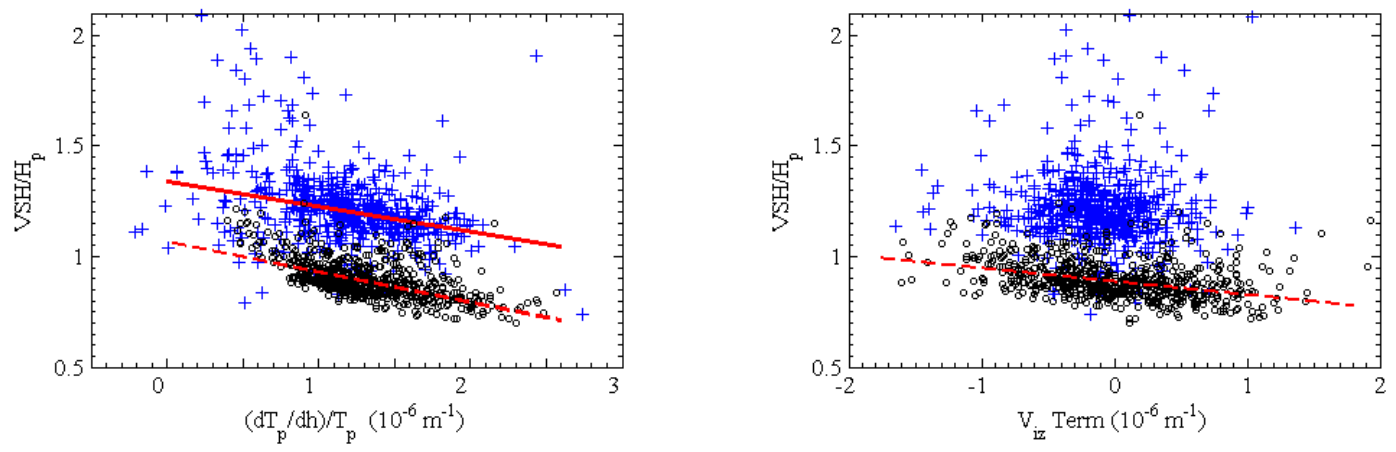

Fig. 4. (Left) Scatterplots of the relative gradient of plasma temperature $\left(\mathrm{d} T_{p} / \mathrm{dh}\right) / T_{p}$ versus VSH/Hp, the ratio of the vertical scale height VSH to plasma scale height $H_{p}$. (Right) Scatterplots of the velocity term in Eq. (2) versus VSH/ $H_{p}$. The curves show the fitting linear trends. In each panel, symbol "+” denotes nighttime values while symbol "o" for daytime values.

to a later time in $H_{p}$. Moreover, there are also peaks in $V_{o}$ and $\left(\mathrm{d} T_{p} / \mathrm{dh}\right) / T_{p}$ over Millstone Hill when the morning peak is present in $H_{p}$. A second peak is quite significant in $\left(\mathrm{d} T_{p} / \mathrm{dh}\right) / T_{p}$ at Millstone Hill, according to Fig. 3. One interesting point is that the nighttime values of $\left(\mathrm{d} T_{p} / \mathrm{dh}\right) / T_{p}$ at Millstone Hill are quite larger than those at Arecibo (Liu et al., 2007).

3.3 The correlation of $\mathrm{VSH} / H_{p}$ versus $\left(\mathrm{d} T_{p} / \mathrm{dh}\right) / T_{p}$ and $V_{i z}$

By considering the vertical drift and ignoring the horizontal gradient in the ionosphere, Liu et al. (2007) derived a relationship between VSH, $H_{p},\left(\mathrm{~d} T_{p} / \mathrm{dh}\right) / T_{p}$, and the vertical diffusion velocity of ions, $\mathrm{W}_{D}$, as

$\frac{1}{\mathrm{VSH}}=\frac{1}{H_{p}}+\frac{m_{i} v_{i n} W_{D}}{k_{b} T_{p}}+\frac{1}{T_{p}} \frac{d T_{p}}{d h}$.

Here $k_{b}$ is the Boltzmann constant, $m_{i}$ the ion mass, and $v_{i n}$ is the collision frequency of ions with neutrals.

Equation (1) suggests that the relationship between VSH and $H_{p}$ is sensitive to the thermal structure and dynamics in the ionosphere. According to Eq. (1), as has been mentioned by Liu et al. (2007), VSH is equal to $H_{p}$, if the topside ionosphere is in a state dominated by diffusive equilibrium $\left(\mathrm{W}_{D}=0\right)$ and the altitude gradient of the thermal structure can be ignored. However, results at both Arecibo and Millstone Hill illustrate that the altitude gradients of plasma temperatures $\left(\mathrm{d} T_{p} / \mathrm{dh}\right)$ are significant in the topside temperature profiles during some local time intervals. Furthermore, besides the contributions from the topside temperature structure, the diffusion process $\left(\mathrm{W}_{D} \neq 0\right)$ can also greatly influence the shape of the topside profiles over Arecibo and Millstone Hill (Luan et al., 2006). Thus, as shown in Fig. 3, VSH will generally deviate from $H_{p}$. As a result, VSH might not be so tightly correlated with the plasma temperature or $H_{p}$, as originally expected.

Utilizing this ISR measurement, we can investigate the relationships between $\mathrm{VSH} / H_{p}$ and $\left(\mathrm{d} T_{p} / \mathrm{dh}\right) / T_{p}$ and the ver- tical velocity of ions over Millstone Hill, as predicted by Eq. (1). The left panel of Fig. 4 shows the correlation between $\mathrm{VSH} / H_{p}$ and $\left(\mathrm{d} T_{p} / \mathrm{dh}\right) / T_{p}$ over Millstone Hill during this experiment. The symbol "+" denotes nighttime values while symbol "o" is used for daytime values. According to the left panel of Fig. 4, the observed tendency of $\mathrm{VSH} / \mathrm{H}_{p}$ versus $\left(\mathrm{d} T_{p} / \mathrm{dh}\right) / T_{p}$ over Millstone Hill during this experiment supports the prediction of Eq. (1); that is, during both the daytime and nighttime, values of $\mathrm{VSH} / H_{p}$ tend to decrease as $\left(\mathrm{d} T_{p} / \mathrm{dh}\right) / T_{p}$ becomes larger. This feature is also found at Arecibo, as reported by Liu et al. (2007). Therefore, we can infer that the topside thermal structure can strongly influence the shape of the topside ionosphere. Further investigation reveals that the contribution of $\left(\mathrm{d} T_{p} / \mathrm{dh}\right) / T_{p}$ is mainly to VSH but not to $H_{p}$ (figure not shown here).

We can also investigate the quantitative relationships between $\mathrm{VSH} / H_{p}$ and the vertical velocity of ions over Millstone Hill. Unfortunately, there is no observed information on neutral wind and the electric field during this measurement. Thus, we cannot deduce diffusion velocity from the line-of-sight velocity $V_{o}$ for this experiment. To avoid introducing artificial information, we directly use $V_{i z}$ as an approximation to $\mathrm{W}_{D}$. The right panel of Fig. 4 illustrates the correlation between $\mathrm{VSH} / H_{p}$ and the term related to the vertical velocity of ions in Eq. (1). Symbol "+" again denotes nighttime values while symbol "o" is used for daytime values. As illustrated in Fig. 4, the daytime values of $\mathrm{VSH} / H_{p}$ have a strong negative tendency with the velocity term of Eq. (1), while this correlation becomes much poorer during the nighttime. We speculate that this scatter is likely due to the day-to-day and local time variabilities in the thermospheric neutral winds and electric fields. Direct observational studies are needed to verify whether or not the relationship between VSH and $H_{p}$ is sensitive to the dynamics in the ionosphere, as predicted in Eq. (1). 


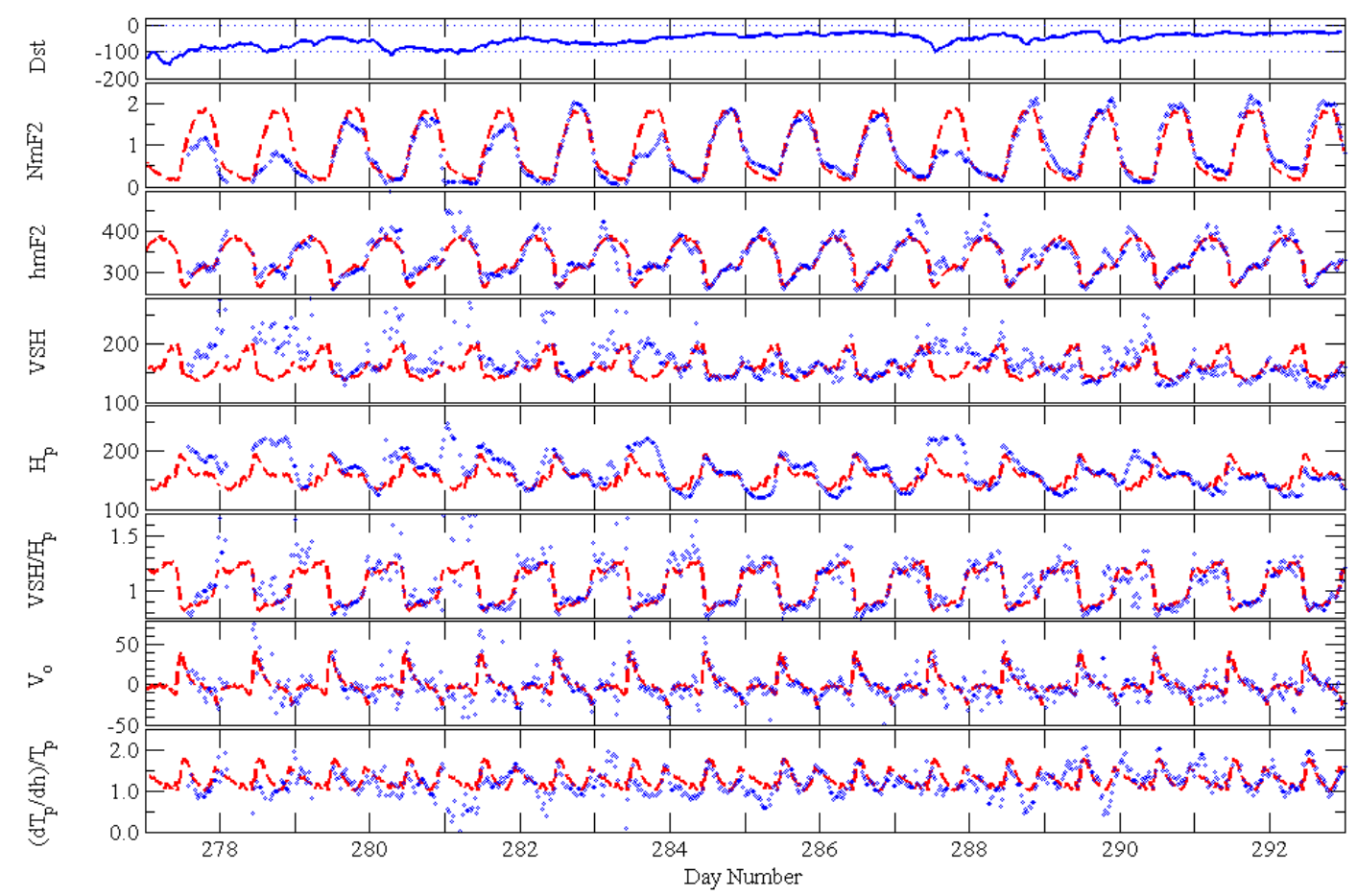

Fig. 5. Geomagnetic index $D_{s t}$ and $N_{m} \mathrm{~F}_{2}, h_{m} \mathrm{~F}_{2}, \mathrm{VSH}, H_{p}, \mathrm{VSH} / H_{p}, V_{o}$, and $\left(\mathrm{d} T_{p} / \mathrm{dh}\right) / T_{p}$ derived from the Millstone Hill ISR experiment during 4 October to 20 October 2002. The dashed curves show the median values, and points for half-hourly averaged values. Here $V_{O}$ represents values around $500 \mathrm{~km}$ in units of $\mathrm{m} / \mathrm{s}, N_{m} \mathrm{~F}_{2}$ is in units of $10^{12} \mathrm{~m}^{-3}$, and $h_{m} \mathrm{~F}_{2}$, VSH and $H_{p}$ in $\mathrm{km}$.

\subsection{The geomagnetic activity effects}

The geomagnetic activity effects on the ionosphere are wellknown to be complicated and stochastic (e.g. Buonsanto, 1999; Kutiev et al., 2005; Liu et al., 2004; Mendillo, 2006). Although there are poor correlations between geomagnetic indices and ionospheric scale heights (Kutiev et al., 2006; Liu et al., 2007; Stankov and Jakowski, 2006b), enhanced geomagnetic activities will increase the variability of the ionospheric scale heights; that is, ionospheric scale heights may greatly deviate from the average pattern under individual disturbed situations, such as the ionogram-derived $H_{m}$ over Wuhan (Liu et al., 2006), ISR profiles derived VSH and $H_{m}$ over Arecibo (Liu et al., 2007), and topside scale height inversed from GPS-TEC (Gulyaeva, 2004).

To illustrate the behavior of the ionosphere over Millstone Hill under geomagnetically quiet and disturbed conditions, Fig. 5 plots the variations of ionospheric parameters during 4 October to 20 October 2002 and Fig. 6 for 21 October to 5 November 2002. Panels in Figs. 5 and 6, from the top to the bottom, are for geomagnetic index $D_{s t}$ and $N_{m} \mathrm{~F}_{2}, h_{m} \mathrm{~F}_{2}$, $\mathrm{VSH}, H_{p}, \mathrm{VSH} / H_{p}, V_{o}$ and $\left(\mathrm{d} T_{p} / \mathrm{dh}\right) / T_{p}$, respectively. The dashed lines denote values serving as the reference level and circle symbols ("o") for half-hourly averaged values.

As seen from Fig. 5, there are appreciable differences in ionospheric parameters under geomagnetically quiet and disturbed conditions. Generally ionospheric parameters follow their median behavior under geomagnetically quiet conditions. Typical examples can be found on day 286 and day 293. During the first active interval, $N_{m} \mathrm{~F}_{2}$ is markedly depressed below its reference level on days 277-279 and on day 281. Apart from a significant evening lift on day 281, $h_{m} \mathrm{~F}_{2}$ presented no significant deviation from the reference level. In contrast, the magnitude of VSH and $H_{p}$ deviated remarkably from the reference level, with enhancements on days 277 and 278. Enhanced variability was presented in $\mathrm{VSH} / H_{p}$ on day 278 and in the early hours of day 279 , in $V_{o}$ near the morning peak on day 278. It is interesting that on day 281, all parameters strongly deviate from their reference levels: $N_{m} \mathrm{~F}_{2}, V_{o}$ and $\left(\mathrm{d} T_{p} / \mathrm{dh}\right) / T_{p}$ were depressed, while $h_{m} \mathrm{~F}_{2}$, VSH, $H_{p}$ and $\mathrm{VSH} / H_{p}$ were greatly enhanced.

During the daytime of day 283, when the geomagnetic activity is moderate and tends to recover to its normal state, there is a remarkable depletion in $N_{m} \mathrm{~F}_{2}$, which can be explained with the storm-time induced changes of neutral compositions and temperature. Accompanying the $N_{m} \mathrm{~F}_{2}$ depletion, a huge enhancement occurred in $h_{m} \mathrm{~F}_{2}$, VSH and $H_{p}$. However, during the daytime huge $N_{m} \mathrm{~F}_{2}$ depletion, no marked change is presented in $h_{m} \mathrm{~F}_{2}$.

During the second active interval, depletions were presented in $V_{o}$ and $\left(\mathrm{d} T_{p} / \mathrm{dh}\right) / T_{p}$. Although $N_{m} \mathrm{~F}_{2}, h_{m} \mathrm{~F}_{2}$ and $H_{p}$ quickly recovered to their normal states, great deviations were still present in $\mathrm{VSH}, \mathrm{VSH} / H_{p}, V_{o}$ and $\left(\mathrm{d} T_{p} / \mathrm{dh}\right) / T_{p}$ for a further 2-3 days. 


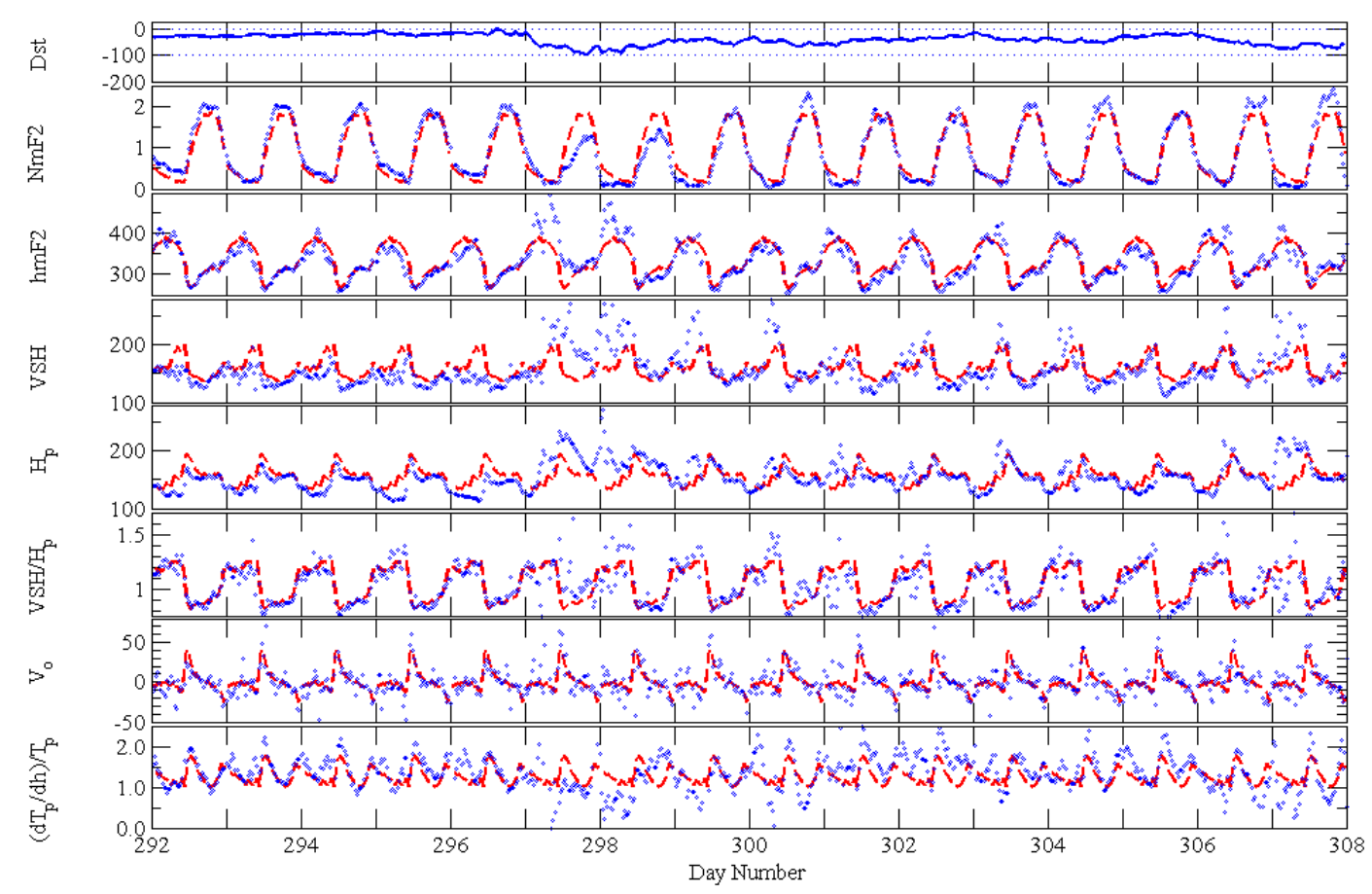

Fig. 6. Same as Fig. 5, but for 21 October to 5 November 2002.

As seen from Fig. 6, during the third active interval, $N_{m} \mathrm{~F}_{2}$ and $\left(\mathrm{d} T_{p} / \mathrm{dh}\right) / T_{p}$ decreased while $h_{m} \mathrm{~F}_{2}, H_{p}$ and VSH increased remarkably on days 297-298. In contrast, another type of effect is found on days 306-307; that is, there is the classic pattern of a dusk effect (e.g. Buonsanto, 1999) in $N_{m} \mathrm{~F}_{2}$, namely a large enhancement seen in $N_{m} \mathrm{~F}_{2}$ during the afternoon and evening hours followed by a negative phase. The dusk effect is believed to be caused by the magnetospheric convection electric fields (e.g. Mendillo, 2006). Accompanying the negative phase in $N_{m} \mathrm{~F}_{2}$ on day 307, VSH and $H_{p}$ were enlarged, and $\mathrm{VSH} / H_{p}$ obviously deviated from the reference level due to the enhanced variability of $\mathrm{VSH} / H_{p}$.

As has been mentioned by Liu et al. (2007), VSH can provide information on the shape of topside electron density profiles. The ionosphere follows the median behavior under geomagnetic quiet conditions. As a result, ionospheric parameters, such as $N_{m} \mathrm{~F}_{2}, h_{m} \mathrm{~F}_{2}, \mathrm{VSH}$ and $H_{p}$, generally follow their median diurnal variations. In contrast, the ionosphere is modified under disturbed conditions. We have noted that a negative phase and dusk effect in $N_{m} \mathrm{~F}_{2}$ are present during this experiment. In the presented case, large differences are detected in VSH and $H_{p}$ under quiet and disturbed magnetic conditions. The variability of the scale heights becomes enhanced under disturbed conditions, indicating that the shape of the topside electron density profiles are appreciably changed due to the storm impacts.

\section{Summary}

Data of $N_{m} \mathrm{~F}_{2}, h_{m} \mathrm{~F}_{2}, \mathrm{VSH}, V_{o}$ and $\left(\mathrm{d} T_{p} / \mathrm{dh}\right) / T_{p}$ and $H_{p}$ deduced from the ISR measurement (4 October to 4 November 2002) at Millstone Hill are analyzed to explore the variations of the ionosphere. The main results are summarized as follows:

1. This statistical analysis identifies a clear average pattern of ionospheric scale heights VSH, $H_{m}$, and $H_{p}$ over Millstone Hill; that is, the diurnal behaviors of the median VSH and $H_{m}$ are found to be greatly similar to each other and are not so tightly correlated with the plasma scale height $H_{p}$ or the plasma temperature.

2. In combining this investigation with that of Luan et al. (2006), evidence exists that both contributions from the temperature structure and diffusion processes can greatly control the shape of the electron density profile and the ratio $\mathrm{VSH} / H_{p}$ in the topside ionosphere over Millstone Hill. Following the theoretical prediction, the values of $\mathrm{VSH} / H_{p}$ tend to decrease as $\left(\mathrm{d} T_{p} / \mathrm{dh}\right) / T_{p}$ becomes larger. This feature is also detected at a lowlatitude station Arecibo (Liu et al., 2007). A similar correlation seems to exist between $\mathrm{VSH} / H_{p}$ and the dynamical processes, while further measurements are still required.

3. Large differences are detected in VSH and $H_{p}$, as well as in $N_{m} \mathrm{~F}_{2}, h_{m} \mathrm{~F}_{2}$, and $\left(\mathrm{d} T_{p} / \mathrm{dh}\right) / T_{p}$ under geomagnetic 
quiet and disturbed conditions. Generally ionospheric parameters follow their median behaviors under geomagnetically quiet conditions, while the variability of the scale heights and other parameters is enhanced under disturbed conditions. In the present case, both VSH and $H_{p}$ increased significantly during storm times, indicating that the geomagnetic activity has a major impact on the behavior of ionospheric scale heights. However, to understand the complexity of the perturbations, more studies are deserved.

Acknowledgements. This study made use of the Millstone Hill ISR data. The Millstone Hill incoherent scatter radar is supported by a cooperative agreement between the National Science Foundation of USA and the Massachusetts Institute of Technology. WDC-C2 provides the geomagnetic indices $D_{s t}, A_{p}$ and AE data. This research was supported by National Natural Science Foundation of China (40725014, 40574071), and National Important Basic Research Project (2006CB806306).

Topical Editor M. Pinnock thanks I. Kutiev and another anonymous referee for their help in evaluating this paper.

\section{References}

Belehaki, A., Marinov, P., Kutiev, I., Jakowski, N., and Stankov, S.: Comparison of the topside ionosphere scale height determined by topside sounders model and bottomside digisonde profiles, Adv. Space Res., 37, 963-966, 2006.

Bilitza, D.: International reference ionosphere 2000, Radio Sci., 36(2), 261-275, 2001.

Bilitza, D., Reinisch, B. W., Radicella, S. M., Pulinets, S., Gulyaeva, T., and Triskova, L.: Improvements of the International reference ionosphere model for the topside electron density profile, Radio Sci., 41, RS5S15, doi:10.1029/2005RS003370, 2006.

Buonsanto, M. J.: Ionospheric storms- A review, Space Sci. Rev., 88, 563-601, 1999.

Gulyaeva, T. L.: Incorporation of topside half peak density anchor point in IRI, Adv. Space Res., 34, 1993-1997, 2004.

Huang, X. and Reinisch, B. W.: Vertical electron profiles from the Digisonde network, Adv. Space Res., 18(6), 121-129, 1996.

Huang, X. and Reinisch, B. W.: Vertical electron content from ionograms in real time, Radio Sci., 36(2), 335-342, 2001.

Isham, B., Tepley, C. A., Sulzer, M. P., Zhou, Q. H., Kelley, M. C., Friedman, J. S., and González, S. A.: Upper atmospheric observations at the Arecibo Observatory: Examples obtained using new capabilities, J. Geophys. Res., 105(A8), 18 609-18 637 , 2000.

Kutiev, I., Watanabe, S., Otsuka, Y., and Saito, A.: Total electron content behavior over Japan during geomagnetic storms, J. Geophys. Res., 110(A1), A01308, doi:10.1029/2004JA010586, 2005.

Kutiev, I. and Marinov, P.: Topside sounder model of scale height and transition height characteristics of the ionosphere, Adv. Space Res., 39(5), 759-766, 2007.

Kutiev, I. S., Marinov, P. G., and Watanabe, S.: Model of topside ionosphere scale height based on topside sounder data, Adv. Space Res., 37, 943-950, 2006.
Lei, J., Liu, L., Wan, W., and Zhang, S.-R.: Variations of electron density based on long-term incoherent scatter radar and ionosonde measurements over Millstone Hill, Radio Sci., 40, RS2008, doi:10.1029/2004RS003106, 2005.

Lei, J., Liu, L., Wan, W., Zhang, S.-R., and van Eyken, A. P.: Comparison of the first long-duration IS experiment measurements over Millstone Hill and EISCAT Svalbard radar with IRI2001, Adv. Space Res., 37, 1102-1107, 2006.

Liu L., Wan, W., Lee, C. C., Ning, B., and Liu, J. Y.: The low latitude ionospheric effects of the April 2000 magnetic storm near the longitude $120^{\circ} \mathrm{E}$, Earth Planets Space, 56, 607-612, 2004.

Liu, L., Wan, W., and Ning, B.: A study of the ionogram derived effective scale height around the ionospheric $h m \mathrm{~F} 2$, Ann. Geophys., 24, 851-860, 2006,

http://www.ann-geophys.net/24/851/2006/.

Liu, L., Le, H., Wan, W., Sulzer, M. P., Lei, J., and Zhang, M.-L.: An analysis of the scale heights in the lower topside ionosphere based on the Arecibo incoherent scatter radar measurements, J. Geophys. Res., 112, A06307, doi:10.1029/2007JA012250, 2007.

Luan, X., Liu, L., Wan, W., Lei, J., Zhang, S.-R., Holt, J. M., and Sulzer, M. P.: A study of the shape of the topside electron density profile derived from incoherent scatter radar measurements over Arecibo and Millstone Hill, Radio Sci., 41, RS4006, doi:10.1029/2005RS003367, 2006.

Mendillo, M.: Storms in the ionosphere: patterns and processes for total electron content, Rev. Geophys., 44, RG4001, doi:10.1029/2005RG000193, 2006.

Oyama, K.-I., Watanabe, S., Su, Y., Takahashi, T., and Hiro, K.: Seasonal, local time, and longitudinal variations of electron temperature at the height of $\sim 600 \mathrm{~km}$ in the low latitude region, Adv. Space Res., 18(6), 269-278, 1996.

Reinisch, B. W. and Huang, X.: Deducing topside profiles and total electron content from bottomside ionograms, Adv. Space Res., 27(1), 23-30, 2004.

Reinisch, B. W., Huang, X., Belehaki, A., Shi, J., Zhang, M., and Ilma, R.: Modeling the IRI topside profile using scale height from ground-based ionosonde measurements, Adv. Space Res., 34, 2026-2031, 2004.

Rishbeth, H. and Garriott, O. K.: Introduction to ionospheric physics, 331 pp., Academic Press, New York, 1969.

Sharma, D. K., Rai, J., Israil, M., and Subrahmanyam, P.: Diurnal, seasonal and longitudinal variations of ionospheric temperatures of the topside F region over the Indian region during solar minimum (1995-1996), J. Atmos. Solar-Terr. Phys., 67, 269-274, 2005.

Stankov, S. M. and Jakowski, N.: Topside plasma scale height retrieved from radio occultation measurements, Adv. Space Res., 37, 958-962, 2006a.

Stankov, S. M. and Jakowski, N.: Topside ionospheric scale height analysis and modeling based on radio occultation measurements, J. Atmos. Solar-Terr. Phys., 68, 134-162, 2006 b.

Stankov, S. M., Jakowski, N., Heise, S., Muhtarov, P., Kutiev, I., and Warnant, R.: A new method for reconstruction of the vertical electron density distribution in the upper ionosphere and plasmasphere, J. Geophys. Res., 108(A5), 1164, doi:10.1029/2002JA009570, 2003.

Tepley, C. A.: Current developments at Arecibo for research in the atmospheric sciences at low latitudes, J. Atmos. Solar-Terr. Phys., 59(13), 1679-1686, 1997. 
Zhang, M.-L., Reinisch, B. W., Shi, J. S., Wu, S., and Wang, X.: Diurnal and seasonal variation of the ionogram-derived scale height at the F2 peak, Adv. Space Res., 37, 967-971, 2006.

Zhang, S.-R., Holt, J. M., Zalucha, A. M., and Amory-Mazaudier, C.: Midlatitude ionospheric plasma temperature climatology and empirical model based on Saint Santin incoherent scatter radar data from 1966 to 1987, J. Geophys. Res., 109, A11311, doi:10.1029/2004JA010709, 2004.
Zhang, S.-R., Holt, J. M., Erickson, P. J., Lind, F. D., Foster, J. C., van Eyken, A. P., Zhang, Y., Paxton, L. J., Rideout, W. C., Goncharenko, L. P., and Campbell, G. R.: October 2002 30-day incoherent scatter radar experiments at Millstone Hill and Svalbard and simultaneous GUVI/TIMED observations, Geophys. Res. Lett., 32, L01108, doi:10.1029/2004GL020732, 2005. 